\title{
Holographic imaging of the complex charge density wave order parameter
}

\author{
Árpád Pásztor $\odot$, Alessandro Scarfato $\odot$, Marcello Spera, Céline Barreteau, Enrico Giannini $\odot$, and Christoph Renner $\odot^{*}$ \\ DQMP, Université de Genève, 24 quai Ernest Ansermet, CH-1211 Geneva 4, Switzerland
}

(Received 11 July 2019; revised manuscript received 30 September 2019; published 19 November 2019)

\begin{abstract}
The charge density wave (CDW) in solids is a collective ground state combining lattice distortions and charge ordering. It is defined by a complex order parameter with an amplitude and a phase. The amplitude and wavelength of the charge modulation are readily accessible to experiment. However, accurate measurements of the corresponding phase are significantly more challenging. Here we combine reciprocal and real space information to map the full complex order parameter based on topographic scanning tunneling microscopy (STM) images. Our technique overcomes limitations of Fourier space based techniques to achieve distinct amplitude and phase images with high spatial resolution. Applying this analysis to transition metal dichalcogenides provides striking evidence that their CDWs consist of three individual unidirectional charge modulations whose ordering vectors are connected by the fundamental rotational symmetry of the crystalline lattice. Spatial variations in the relative phases of these three modulations account for the different CDW contrasts often observed in STM topographic images. Phase images further reveal topological defects and discommensurations, a singularity predicted by theory for a nearly commensurate CDW. Such precise real space mapping of the complex order parameter provides a powerful tool for a deeper understanding of the CDW ground state whose formation mechanisms remain largely unclear.
\end{abstract}

DOI: 10.1103/PhysRevResearch.1.033114

\section{INTRODUCTION}

The spatially averaged intensity of the CDW order parameter is usually accessed by scattering techniques sensitive to the local lattice distortions, or electron spectroscopy and transport measurements sensitive to changes in the band structure due to the opening of the CDW gap. Detecting the phase has been traditionally limited to dynamic experiments (for good reviews, see, e.g., Refs. [1,2]). More recently, different strategies have been followed to access phase information from spectroscopic [3,4] and topographic [5] local probe tunneling experiments as well as from the periodic distortion of the atomic lattice measured by transmission electron microscopy $[6,7]$. However, there are many difficulties, e.g., extracting phase information from reciprocal space data tends to suffer from phase wrapping and fundamental numerical difficulties of finite sampling (see Supplemental Material Sec. III [8]). Unwrapping the phase in these cases leads to singularities that can easily be mistaken for physical phase variations that actually do not exist and it can also obscure real features, for instance, constant phase domains in $1 \mathrm{~T}-\mathrm{Cu}_{x} \mathrm{TiSe}_{2}$. Combining reciprocal and real space scanning tunneling microscopy (STM) data to extract the full complex CDW order parameter enables us to overcome these limitations.

\footnotetext{
*Christoph.Renner@unige.ch

Published by the American Physical Society under the terms of the Creative Commons Attribution 4.0 International license. Further distribution of this work must maintain attribution to the author(s) and the published article's title, journal citation, and DOI.
}

The CDW state is characterized by a real space periodic modulation of the charge density [9]:

$$
\delta \rho(\mathbf{r})=\rho(\mathbf{r})-\rho_{0}=A \cdot \cos (\mathbf{q} \cdot \mathbf{r}+\varphi)=\operatorname{Re}\left(\Psi e^{i \mathbf{q} \cdot \mathbf{r}}\right),
$$

where $\rho_{0}$ is the constant background charge density in the metallic state, $\mathbf{q}$ is the ordering vector, and $A$ is the modulation amplitude. The order parameter of the CDW state is a complex quantity: $\Psi=A \cdot e^{i \varphi}$. In general, one expects its amplitude and phase to spatially vary $[A=A(\mathbf{r})$ and $\varphi=\varphi(\mathbf{r})]$, leading to collective excitations (amplitudons and phasons, respectively) and defects (domain walls, vortices, discommensurations, etc.) in the CDW condensate.

In this study, we focus on the complex CDW order parameter in three transition metal dichalcogenides (TMDCs) $M \mathrm{Se}_{2}$. They are layered materials where each slab is composed of a triangular metal layer $M(\mathrm{Ti}, \mathrm{Nb}$, and $\mathrm{V}$ in the present study) sandwiched between two triangular selenium sheets. The layer stacking is maintained by weak van der Waals (vdW) forces allowing facile cleaving between the slabs exposing a triangular Se layer to the surface. In $1 \mathrm{~T}-\mathrm{Cu}_{x} \mathrm{TiSe}_{2}$, copper atoms intercalate on the octahedral site in the vdW gap between the $\mathrm{TiSe}_{2}$ slabs [10]. 1T-Cu $\mathrm{Cu}_{x} \mathrm{TiSe}_{2}$ and $1 \mathrm{~T}-\mathrm{VSe}_{2}$ host a short-range $2 a \times 2 a$ and a commensurate $4 a \times 4 a \mathrm{CDW}$ within the basal plane [10-13], respectively, while $2 \mathrm{H}-\mathrm{NbSe}_{2}$ has been shown to develop a slightly incommensurate $(3+\delta) a \times(3+\delta) a$ CDW modulation [14], with $\delta \approx$ 0.03-0.08 depending on temperature.

All three compounds studied here have a crystal structure with a three-fold symmetry in the ab-plane, regardless of their specific polytype (1T or $2 \mathrm{H})$. The crystal symmetry implies that if there exists an ordering vector $\mathbf{q}_{1}$ for which the formation of a CDW is favorable in the ab-plane, there are two other momentum vectors $\mathbf{q}_{2}$ and $\mathbf{q}_{3}$ for which the CDW formation 
is equally favorable. These three $q$ vectors are connected by a three-fold $2 \pi / 3$ rotation symmetry and are often referred to as the three components of the tridirectional CDW. The total charge density modulation in space can be expressed as the sum of these three charge density modulations

$\delta \rho(\mathbf{r})=\sum_{n=1}^{3} A_{n}(\mathbf{r}) \cdot \cos \left(\mathbf{q}_{n} \cdot \mathbf{r}+\varphi_{n}(\mathbf{r})=\sum_{n=1}^{3} \operatorname{Re}\left(\Psi_{n} e^{i \mathbf{q}_{n} \cdot \mathbf{r}}\right)\right.$,

where $\Psi_{n}=A_{n}(\mathbf{r}) e^{i \varphi_{n}(\mathbf{r})}$ is the order parameter of the $n$th direction and $\mathbf{q}_{n}, \varphi_{n}(\mathbf{r})$, and $A_{n}(\mathbf{r})$ are the corresponding ordering vectors, phase shifts, and local amplitudes. Hereafter and following the proposition of McMillan [15], we refer to the CDW modulations in the three $\mathbf{q}_{n}$ directions as the three coexisting CDWs, a denomination fully justified by our experimental findings in the following sections.

\section{METHODS}

Single crystals of $M \mathrm{Se}_{2}\left(M=\mathrm{Cu}_{0.02} \mathrm{Ti}, \mathrm{Nb}, \mathrm{V}\right)$ were grown via the chemical vapor transport method using iodine as the transport agent and cleaved in situ at room temperature. STM experiments were done in UHV (base pressure below $2 \times 10^{-10}$ mbar) using tips either electrochemically etched from an annealed tungsten wire or mechanically cut from a PtIr wire, with similar results. All tips were conditioned in situ on cleaned and reconstructed $\mathrm{Au}(111)$ or $\mathrm{Ag}(111)$ single crystals. The bias voltage was applied to the sample. STM images were recorded with at least $20 \mathrm{pixel} / \mathrm{nm}$ resolution. More details of the fitting procedure is given in Supplemental Material Sec. II [8].

\section{REAL SPACE CHARGE DENSITY WAVE PATTERN}

A rapid survey of the literature reveals that the $\mathrm{CDW}$ contrast in a given material may vary significantly from image to image in distinct STM studies, even in the absence of any structural defects. While in several cases this can be explained by changing experimental conditions [8,16-18], either extrinsic, e.g., a change in the tip state, or intrinsic, e.g., bias dependence, we find compelling evidence of contrast variations within the same image which are real CDW features and not the consequence of particular experimental conditions. In Figs. 1(a), 1(c) and 1(d) we highlight two adjacent regions with a different contrast in the same STM micrograph. As we show in the following, these two contrasts can be reproduced straightforwardly by tuning the relative phases of the three CDWs discussed above. To quantify the changing configurations, we introduce a dephasing parameter $\Theta$ defined as the sum of the three individual CDW phases $\varphi_{n}$ modulo $2 \pi$ :

$$
\Theta=\left(\sum_{n=1}^{3} \varphi_{n}\right) \bmod 2 \pi .
$$

Theta is a robust parameter uniquely defining a particular CDW STM topographic imaging contrast alone, i.e., without the underlying atomic lattice (see Supplemental Material Sec. I [8] for a geometrical interpretation of the dephasing parameter). It is completely independent of the reference frame,

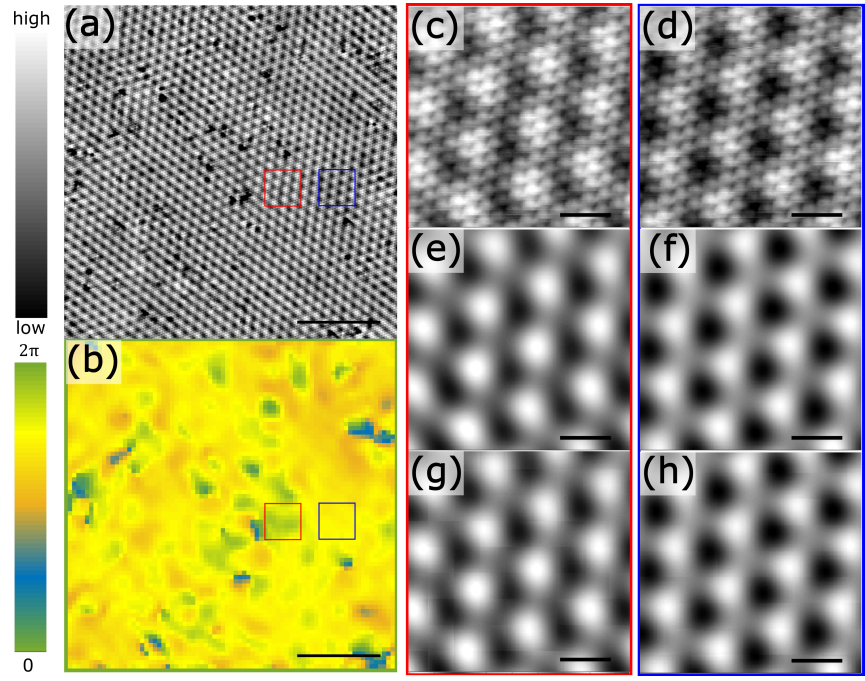

FIG. 1. Real space CDW pattern and dephasing parameter (a) High resolution STM micrograph $\left(V_{\text {bias }}=-100 \mathrm{mV}, I_{t}=1 \mathrm{nA}\right)$ of an in situ cleaved $1 \mathrm{~T}-\mathrm{VSe}_{2}$ surface at $40 \mathrm{~K}$. The atomic lattice and the CDW modulation are clearly resolved. (b) Spatial variation of the dephasing parameter as determined by the fitting procedure. The areas marked by red and blue squares have distinct dephasing parameters corresponding to the different appearances of the CDW. (c) and (d) are magnified areas corresponding to the red and blue squares in (a) respectively. They are connected by single scan lines, the different contrasts can thus not be blamed on different tip state configurations. (e) and (f) are zooms into the large Fourier filtered image of the CDWs at the same location as shown in (c) and (d) respectively. $(\mathrm{g})$ and $(\mathrm{h})$ are reconstructed images from the mosaics of the fitting procedure, in excellent agreement with (e) and (f). The topographic images are all plotted with the exact same color scale. Scale bars: $10 \mathrm{~nm}$ in (a) and (b) and $1 \mathrm{~nm}$ in (c)-(h).

contrary to the individual phases $\varphi_{n}$ of each unidirectional CDW which depend on the selected reference frame.

To determine the phase $\varphi_{n}$ of each $\Psi_{n}$ required to compute the dephasing parameter, we developed a procedure based on the local $\mathbf{q}_{n}$-specific fitting of the real space CDW modulation measured by STM. We locally describe each CDW by a two-dimensional plane wave function $f_{n}(\mathbf{r})=$ $A_{n}(\mathbf{r}) \cos \left[\frac{2 \pi}{\lambda_{n}} \hat{\mathbf{q}}_{n} \cdot \mathbf{r}+\varphi_{n}(\mathbf{r})\right]$, where $\hat{\mathbf{q}}_{n}$ is a unit vector in the direction of the $n$th ordering vector and $\lambda_{n}$ is the CDW wavelength. We consider a model where we allow the amplitude $A_{n}(\mathbf{r})$ and the phase $\varphi_{n}(\mathbf{r})$ for each direction $(n=1,2,3)$ to vary spatially. The result is a complete local characterization of the CDW modulation.

To illustrate the fitting procedure described in more detail in Supplemental Material Sec. II [8], we apply it to a largescale STM image of an in situ cleaved $1 \mathrm{~T}-\mathrm{VSe}_{2}$ single crystal measured at $40 \mathrm{~K}$ (Fig. 1). The atomic and the $4 a \times 4 a \mathrm{CDW}$ modulations are clearly resolved in the micrograph [Fig. 1(a)]. The red and blue squares, magnified in Figs. 1(c) and 1(d), highlight two adjacent regions with different CDW contrasts. This image was acquired from top to bottom, with the fast scan direction running horizontally. The two highlighted regions are thus connected through continuous scan lines and therefore imaged with exactly the same tip at the same set point. Consequently, the distinct appearances of the CDW in 


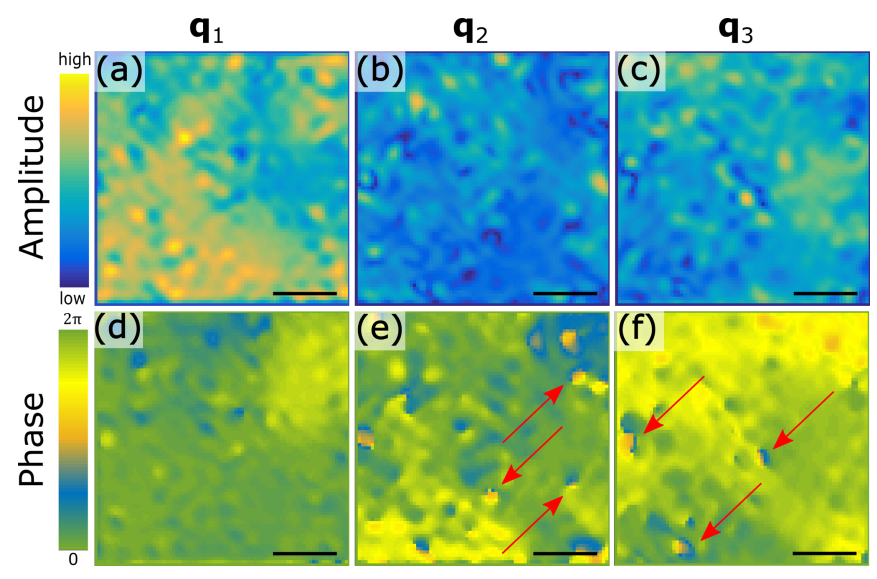

FIG. 2. Coexisting charge density waves. Mapping of the full complex order parameter in $1 \mathrm{~T}-\mathrm{VSe}_{2}$ for the same surface area as in Fig. 1(a). (a)-(c) The local amplitude of the CDWs in each direction $\left(\mathbf{q}_{1}, \mathbf{q}_{2}, \mathbf{q}_{3}\right.$ respectively) and (d)-(f) the corresponding local phase. For easier comparison of the spatial evolution of the local phases of each $\mathbf{q}_{n}$, they are referenced to the lower left corner, whose phase is arbitrarily set to zero. Scale bar: $10 \mathrm{~nm}$. Red arrows are indicating tightly bound vortex-antivortex pairs.

these two regions cannot be imputed to changing tunneling conditions. Magnifying the same two regions of the Fourier filtered image of Fig. 1(a) clearly shows the CDW origin of the changing contrast [Figs. 1(e) and 1(f)]. Indeed, applying our fitting procedure allows us to perfectly reproduce the experimental CDW contrast [Figs. 1(g) and 1(h)] characterized by two distinct dephasing parameters $\Theta$ displayed in Fig. 1(b). Running the fitting procedure on a dense grid of slightly overlapping small windows spanning the entire field of view of Fig. 1(a) allows us to reconstruct the complete spatial structure of the dephasing parameter [Fig. 1(b)] and the CDW pattern. We find that the changing contrast over the entire scan area is fully reproduced by a specific local dephasing parameter with a remarkable match between the experimental and the calculated contrasts as exemplified in Figs. 1(e)-1(h).

\section{COEXISTING CHARGE DENSITY WAVES}

So far, we have established the ability of our model to account for the variety of CDW contrasts observed in STM micrographs of a given material using a single well-defined dephasing parameter. We now advance a step further in the quantitative analysis to discuss the individual amplitudes and phases of each of the three CDWs as a function of position. The spatially resolved amplitudes $A_{n}(\mathbf{r})$ and the corresponding local phases $\varphi_{n}(\mathbf{r})$ for each $\mathbf{q}_{n}$, over the same field of view as Fig. 1(a), are shown in Figs. 2(a)-2(c) and in Figs. 2(d)2(f), respectively. A remarkable aspect of this dataset is the absence of any obvious spatial correlation between the amplitude and phase images of the different CDWs. Each map displays its own features, meaning the amplitude and the phase of each CDW can vary at some location, even abruptly, regardless of the other two CDWs whose amplitudes and phases can remain constant and featureless at that same position. This clearly shows that these three CDWs develop their individual local complex order parameters, albeit being indistinguishable in the sense that they are connected by symmetry. We find a very similar behavior in $1 \mathrm{~T}-\mathrm{Cu}_{0.02} \mathrm{TiSe}_{2}$ and $2 \mathrm{H}-\mathrm{NbSe}_{2}$ (see Suppl. Figs. 7 and 8 [8]), supporting the idea that the total charge density modulation in these TMDCs consists of three coexisting CDWs. Note that although we find three rather independent $\Psi_{n}$ in the examples discussed here, interactions mediated by the atomic lattice are possible. For example, pinning by crystalline defects or impurities can distort the charge modulations $[19,20]$ and introduce correlations between the three CDWs.

\section{SINGULARITIES OF THE COMPLEX ORDER PARAMETER}

The ability to determine the local amplitude and phase of each CDW enables a much more thorough characterization of the CDW ground state in real space. Beyond explaining the variety of STM imaging contrasts and demonstrating the individual nature of the three CDWs developing in TMDCs, our fitting procedure reveals several intrinsic CDW properties. Of particular interest are singularities in the amplitude and phase, such as domain walls and topological defects (vortices). They are important to understanding the interplay of ordered electronic phases [3,21]. For example, the model analysis discussed here provides a unique - quantitative, unbiased, and automated-contrast mechanism to identify and locate domain walls, which have been proposed to promote superconductivity [22,23]. We now focus on three selected CDW features to illustrate the augmented experimental phase space accessible by fitting the complex order parameter in real space.

\section{A. Phase domain walls in $\mathrm{Cu}_{0.02} \mathrm{TiSe}_{2}$}

The first example is a detailed analysis of $\pi$-phase shift CDW domain walls ( $\pi$ DWs) developing in $\mathrm{TiSe}_{2}$ when intercalating either $\mathrm{Cu}$ [10] or large amounts of Ti [24]. These $\pi$ DWs are usually identified in STM micrographs by eye, seeking for one atomic row shifts in the characteristic sequence of bright and dark atoms of the $2 a \times 2 a$ CDW. Such domains can be easily found in Fig. 3(a). Their topographic structure is magnified in Figs. 3(b) and 3(c) corresponding to the red and blue outlined regions in Fig. 3(a). The real space CDW modulation in these two regions highlighted in the corresponding filtered images [Figs. 3(d) and 3(e)] is perfectly reproduced by our fitting procedure in Figs. 3(f) and 3(g). Most instructive are the local phases of the three independent CDWs involved in the above fitting procedure [Fig. 3(i)-3(k)]. Each phase is essentially constant within any given domain and changes abruptly at the domain wall. Our analysis reveals that the phase shift at the domain walls is not always exactly $\pi$, as shown by the polar histograms in Figs. 3(1)-3(o). Such deviations cannot be detected by a visual inspection without the quantitative phase analysis proposed here, because the same atoms remain the brighter ones within each domain up to a phase shift close to $\pi / 4$ (see Supplemental Material Sec. IV and Suppl. Fig. 6 [8]). Our holographic analysis further reveals these $\pi$ DWs to be one-dimensional line singularities along which the CDW order parameter amplitude is suppressed [Suppl. Figs. 7(a)-7(c) [8]]. Further supporting the collapse of the CDW state at the $\pi$ DWs are the low values 

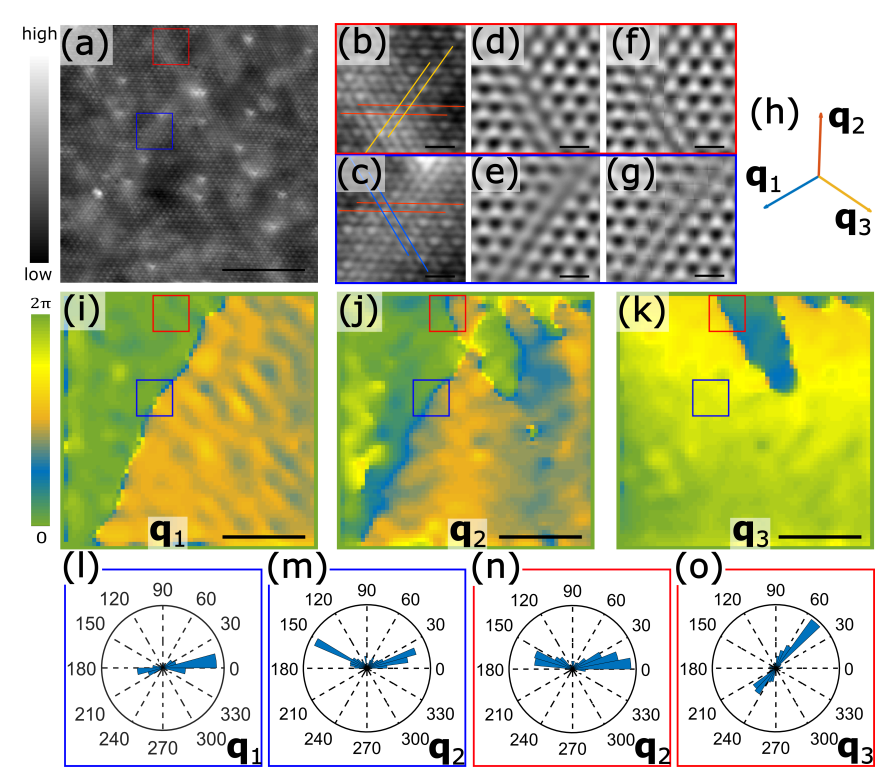

FIG. 3. Phase domains of the CDW in $1 \mathrm{~T}-\mathrm{Cu}_{0.02} \mathrm{TiSe}_{2}$ at $1.2 \mathrm{~K}$. (a) High resolution STM micrograph of an in situ cleaved surface $\left(V_{\text {bias }}=-200 \mathrm{mV}, I_{t}=100 \mathrm{pA}\right)$. (b) and (c) are selected magnified areas from (a) at locations marked by red and blue squares, respectively. Blue $\left(\mathbf{q}_{1}\right)$, orange $\left(\mathbf{q}_{2}\right)$, and yellow $\left(\mathbf{q}_{3}\right)$ parallel lines highlight the shifted CDW modulations for each direction. (d) and (e) show the Fourier filtered CDW seen in (b) and (c). (f) and (g) are reconstructed images from the mosaics of the fitting procedure. Note the excellent agreement with (d) and (e). (h) Vectors representing the CDW $q$ vectors. (i)-(k) are the fitted phase images for the three directions $\left(\mathbf{q}_{1}, \mathbf{q}_{2}, \mathbf{q}_{3}\right)$. The red and blue squares mark the same areas as in (a). In the red region, $\mathbf{q}_{2}$ and $\mathbf{q}_{3}$ show a phase shift [see (b)]. This is very well reflected in (i)-(k): within the red region $\mathbf{q}_{1}$ is homogeneous (no shift) while the color of $\mathbf{q}_{2}$ and $\mathbf{q}_{3}$ changes abruptly at the DW. Similarly, in the blue region $\mathbf{q}_{1}$ and $\mathbf{q}_{2}$ shift, while $\mathbf{q}_{3}$ does not [see (c)]. This is very well shown in (i)-(k): $\mathbf{q}_{1}$ and $\mathbf{q}_{2}$ show an abrupt color change at the DW in the blue region, but $\mathbf{q}_{3}$ remains uniform. (1)-(o) are polar histograms of the CDW phase (in degrees) corresponding to (l) blue area in (i); (m) blue area in (j); (n) red area in (j); and (o) red area in (k). Scale bars $=10 \mathrm{~nm}$ in (a) and (i) $-(\mathrm{k})$; scale bars $=1 \mathrm{~nm}$ in (b) $-(\mathrm{g})$.

of the $\mathrm{R}^{2}$ maps along the domain walls [Suppl. Figs. 7(g)-7(i) [8]], indicating significant deviations from the simple fitted plane wave model at these locations.

In general, the $\mathrm{R}^{2}$ maps provide an insightful direct and local measure of the adequacy of the plane wave model. Note that $\mathrm{R}^{2}$ is not systematically poor at domain walls or near defects (Suppl. Figs. 7 and 8 [8]), which means they are not all identical. Moreover, $\mathrm{R}^{2}$ can be low at a given defect for one or two of the CDWs, but remain perfectly robust (i.e., $\left.\mathrm{R}^{2} \approx 1\right)$ at the same defect in the other CDWs. $\mathrm{R}^{2}$ maps thus reveal real features in the CDWs emphasizing internal physical structures.

\section{B. Discommensurations}

As a second example, we address discommensurations (DCs), a particular type of defect associated with nearly commensurate CDWs (NC-CDWs) found for example in $2 \mathrm{H}-\mathrm{NbSe}_{2}$ or $2 \mathrm{H}-\mathrm{TaSe}_{2}$. Discommensurations were first
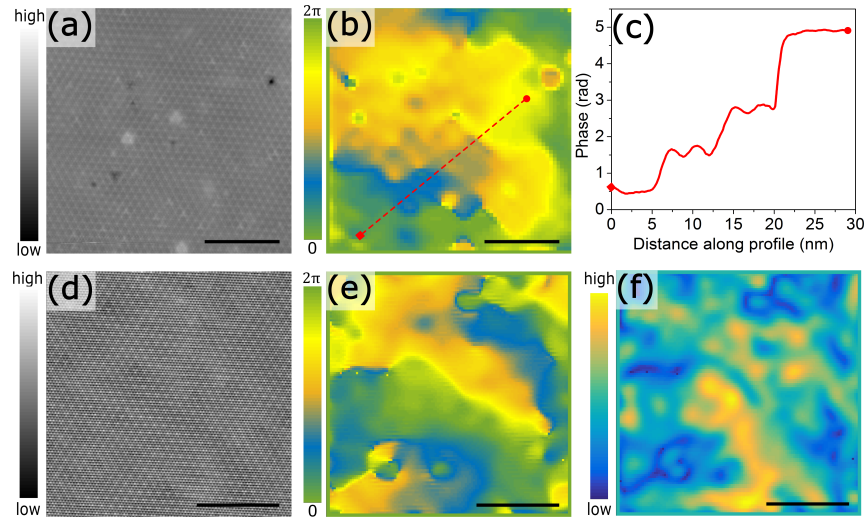

FIG. 4. STM imaging of crystalline defects, CDW discommensurations and singularities in $2 \mathrm{H}-\mathrm{NbSe}_{2}$. (a) STM micrograph with atomic defects. (b) Phase map of the corresponding $\mathbf{q}_{2} \mathrm{CDW}$. (c) Phase profile extracted from the diamond to the circle along the red dashed line in (b). (d) STM micrograph of a defect-free region on the same sample. (e) Phase and (f) amplitude maps of the corresponding $\mathbf{q}_{2}$ CDW. Both STM micrographs were measured at $1.2 \mathrm{~K}$ on an in situ cleaved surface. Tunneling parameters: $V_{\text {bias }}=$ $100 \mathrm{mV}, I_{t}=100 \mathrm{pA}$. Scale bars $=10 \mathrm{~nm}$.

proposed in the seminal work of McMillan [15] and searched for, e.g, in the NC-CDW phase of $1 \mathrm{~T}-\mathrm{TaS}_{2}$ [25] and in the charge ordered phase of cuprate superconductors [26]. DCs are domain walls where the order parameter phase is changing rapidly between small phase locked regions, which allow the CDW system to lower its energy. Our holographic analysis reveals precisely such a phase texture in the CDW images acquired on in situ cleaved surfaces of $2 \mathrm{H}-\mathrm{NbSe}_{2}$ [Fig. 4(a)]. Figure $4(\mathrm{~b})$ is a phase map of one of the three CDWs in the same field of view as Fig. 4(a). It reveals areas of rather constant phase separated by nanometer-sized regions (domain walls) where the phase is changing rapidly. This staircase structure is best seen in a phase profile [Fig. 4(c)] taken along the red dashed line in Fig. 4(b) which perfectly mimics the prediction by McMillan [15]. The phase-locked region sizes and locations are uncorrelated among the CDW components [Suppl. Figs. 8(d)- 8(f) [8]], which confirms their individual nature. We stress that the DC domain walls observed here for the NC-CDW of $2 \mathrm{H}-\mathrm{NbSe}_{2}$ are completely different from the $\pi$ DWs discussed above for $1 \mathrm{~T}-\mathrm{Cu}_{0.02} \mathrm{TiSe}_{2}$. The order parameter amplitude remains essentially unchanged along a DC [Suppl. Figs. 8(a)-8(c) [8]], whereas it is vanishing along the $\pi$ DWs [Suppl. Figs. 7(a)-7(c) [8]], underlining the different nature and origin of these two CDW defects. While $\pi \mathrm{DWs}$ can be readily seen in topographic STM images, DCs can only be detected using our holographic fitting scheme. Note that in order to detect DCs, one has to control the phase wrapping in the fitting procedure. Otherwise, cumulative errors introduce a strong artificial phase gradient in the phase-locked domains delimited by the DCs, making them essentially undetectable (see Suppl. Fig. 5 [8]).

\section{Topological defects}

The final example are topological defects (TDs) which are defined as singularities in the order parameter that cannot be 
(a)
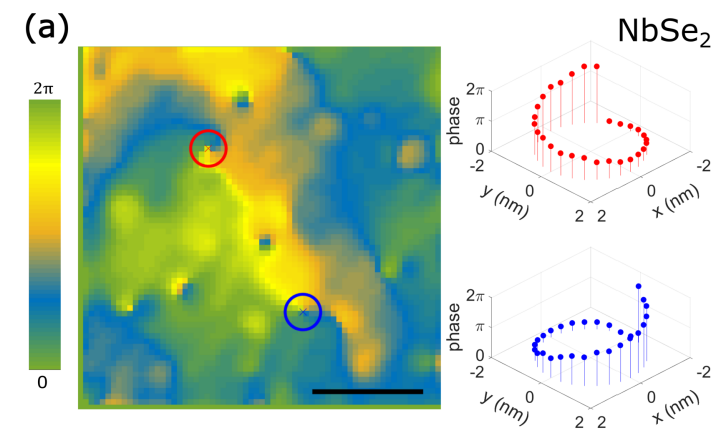

(b)
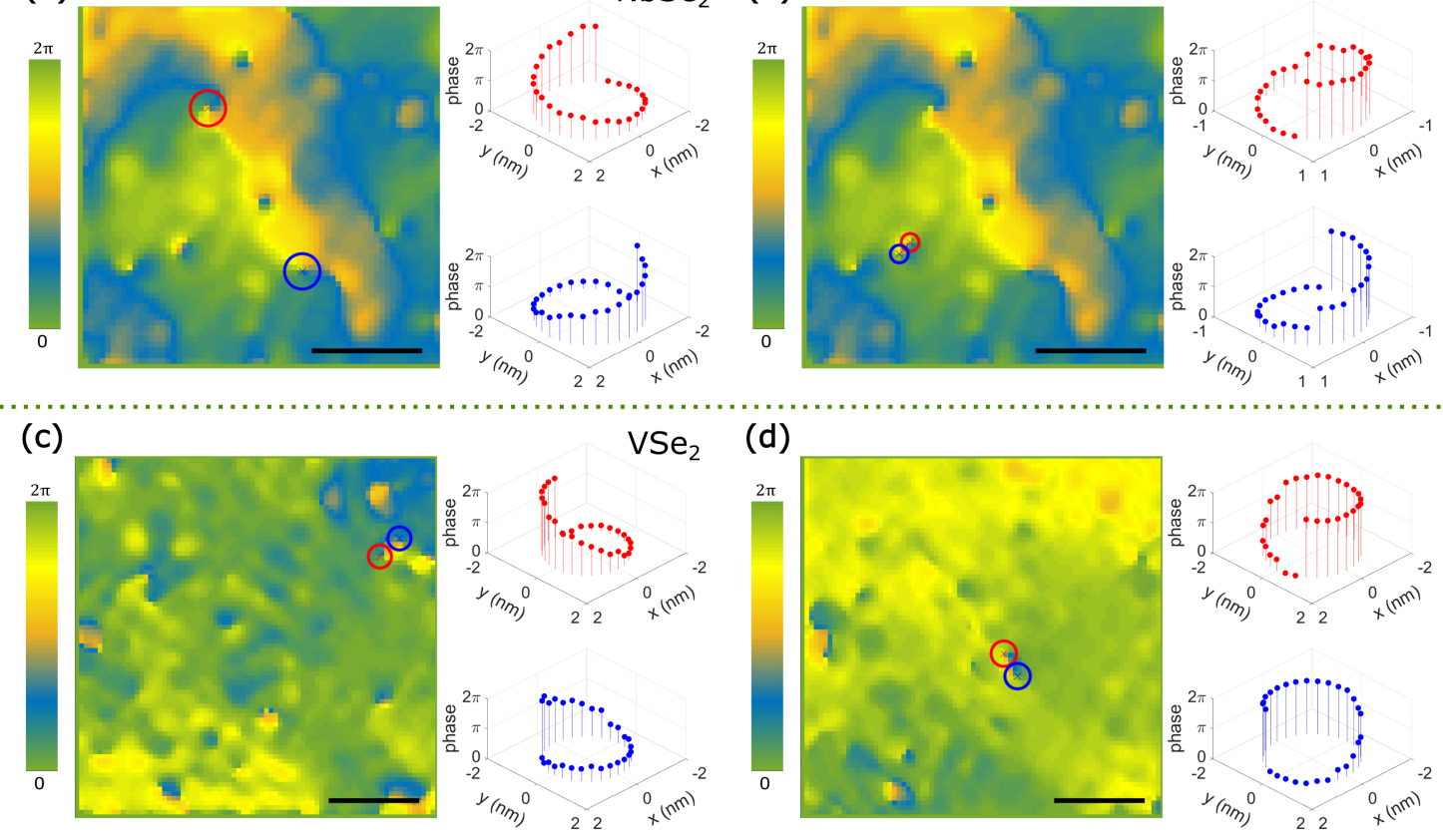

(d)

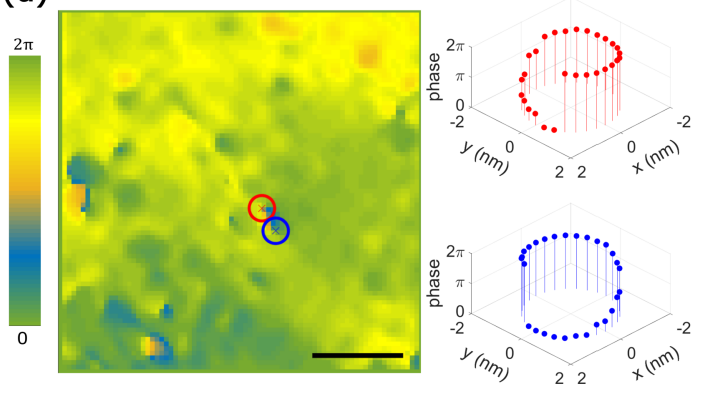

FIG. 5. CDW phase mapping around topological defects. (a) Phase profile around a weakly bound vortex-antivortex pair in NbSe $\mathrm{N}_{2}$ and (b)-(d) around strongly bound pairs in $\mathrm{NbSe}_{2}$ and $\mathrm{VSe}_{2}$. The red and blue profiles were taken along the circles with the corresponding color in each phase map to their left. Note the opposite winding polarities of the vortices (in blue) and antivortices (in red). The corresponding STM micrographs for $\mathrm{NbSe}_{2}$ and $\mathrm{VSe}_{2}$ are shown in Figs. 4(a) and 1(a), respectively. Scale bar $=10 \mathrm{~nm}$.

annealed without introducing other singularities outside of an arbitrarily small environment of the TDs [27]. They are characterized by a topological invariant, the winding number, which is conserved under any continuous deformation of the order parameter field. They correspond to points in a $2 \mathrm{D}$ landscape around which the total phase of the order parameter winds by an integer multiple of $2 \pi$. By analogy to vortices in a superconductor, they are called vortices and antivortices depending on the winding polarity (see Fig. 5). Such defects appear in the phase images of both $1 \mathrm{~T}-\mathrm{VSe}_{2}$ and $2 \mathrm{H}-\mathrm{NbSe}_{2}$. We observe tightly bound vortex-antivortex pairs, as pointed out by red arrows in Figs. 2(e) and 2(f) measured on 1T-VSe and well separated vortex-antivortex pairs, as pointed out by white arrows in Suppl. Figs. 8(d)- 8(f) [8] measured on $2 \mathrm{H}-\mathrm{NbSe}_{2}$. The TD landscape is different for each of the three CDWs in a given STM image, again supporting the conclusion that the CDW consists of three individual charge modulations.

\section{Structural and CDW defects}

Crystalline defects and impurities certainly play a role in the amplitude and phase landscape of the CDW $[19,20]$ and in the nucleation near the phase transition [28]. The holographic analysis we propose here unveils more subtle aspects of this interplay. A revealing insight is that domain walls and TDs can develop independently in any of the three CDWs as clearly exemplified by the distinct domain patterns of the three CDWs of $1 \mathrm{~T}-\mathrm{Cu}_{0.02} \mathrm{TiSe}_{2}$ in Figs. 3(i)-3(k). An additional striking manifestation of the coexistence of individual charge orders, highlighted by our holographic analysis, is that a single atom defect can trigger a topological defect in the phase of one CDW, while only producing a smooth phase variation for another one (Fig. 2 and Suppl. Fig. 8 [8]). While CDW defects are expected in the presence of structural defects, the holographic analysis discussed here reveals that the CDW order parameter landscape [Figs. 4(e) and 4(f)] can be remarkably inhomogeneous on a $2 \mathrm{H}-\mathrm{NbSe}_{2}$ surface with no obvious structural defects [Fig. 4(d)]. This is likely the result of discommensurations not readily detectable in the topographic STM images. It may also reflect 3D CDW correlations with defects below the surface not apparent in the STM micrograph. More generally, Figs. 4(d)-4(f) suggest the ability of the CDW to develop a spatial structure essentially independent of the supporting crystalline lattice.

\section{CONCLUSION}

The holographic imaging of the local complex CDW order parameter with a spatial resolution of the order of the CDW wavelength offers remarkable opportunities to uncover physical details of the CDW state. We find clear evidence for the coexistence of three individual CDWs in transition metal dichalcogenides with a threefold symmetric band structure in the ab-plane, each developing a distinct order parameter landscape and a great variety of features. Unambiguous phase images contribute additional insight into the CDW $\pi$ DWs reported in $1 \mathrm{~T}-\mathrm{Cu}_{0.02} \mathrm{TiSe}_{2}$, demonstrating that the phase shifts of the CDWs can slightly deviate from $\pi$. Mastering the phase fitting procedure reveals constant phase domains and provides valuable insight into nearly commensurate $\mathrm{CDWs}$, with real space phase images of individual discommensurations in $2 \mathrm{H}-\mathrm{NbSe}_{2}$ which were predicted long ago by theory. We 
expect significant contributions to the deeper understanding of charge density waves, including their formation mechanism and their interplay with other correlated electron ground states (e.g., superconductivity), from the expanded parameter space accessible through the high-resolution real space mapping of the complex CDW order parameter.

\section{ACKNOWLEDGMENTS}

We thank A. Guipet and G. Manfrini for their technical support in the STM laboratories. This project was supported by Div. 2 (Grant No. 182652) and Sinergia (Grant No. 147607) of the Swiss National Science Foundation.
[1] P. Monceau, Electronic crystals: An experimental overview, Adv. Phys. 61, 325 (2012).

[2] G. Grüner, The dynamics of charge-density waves, Rev. Mod. Phys. 60, 1129 (1988).

[3] A. Mesaros, K. Fujita, H. Eisaki, S. Uchida, J. C. Davis, S. Sachdev, J. Zaanen, M. J. Lawler, and E.-A. Kim, Topological defects coupling smectic modulations to intra-unit-cell nematicity in cuprates, Science 333, 426 (2011).

[4] M. H. Hamidian, S. D. Edkins, C. K. Kim, J. C. Davis, A. P. Mackenzie, H. Eisaki, S. Uchida, M. J. Lawler, E. A. Kim, S. Sachdev, and K. Fujita, Atomic-scale electronic structure of the cuprate d-symmetry form factor density wave state, Nat. Phys. 12, 150 (2015).

[5] J. Okamoto, C. J. Arguello, E. P. Rosenthal, A. N. Pasupathy, and A. J. Millis, Experimental Evidence for a Bragg Glass Density Wave Phase in a Transition-Metal Dichalcogenide, Phys. Rev. Lett. 114, 026802 (2015).

[6] B. H. Savitzky, I. El Baggari, A. S. Admasu, J. Kim, S.-W. Cheong, R. Hovden, and L. F. Kourkoutis, Bending and breaking of stripes in a charge ordered manganite, Nat. Commun. 8, 1883 (2017).

[7] I. El Baggari, B. H. Savitzky, A. S. Admasu, J. Kim, S.-W. Cheong, R. Hovden, and L. F. Kourkoutis, Nature and evolution of incommensurate charge order in manganites visualized with cryogenic scanning transmission electron microscopy, Proc. Natl. Acad. Sci. USA 115, 1445 (2018).

[8] See Supplemental Material at http://link.aps.org/supplemental/ 10.1103/PhysRevResearch.1.033114 for a more detailed description of the dephasing parameter, more details of the fitting procedure and Fourier space based phase retrieval techniques, and additional insights into CDW defects.

[9] G. Grüner, Density Waves in Solids (Westview, Boulder, CO, 2000).

[10] A. M. Novello, M. Spera, A. Scarfato, A. Ubaldini, E. Giannini, D. R. Bowler, and C. Renner, Stripe and Short Range Order in the Charge Density Wave of $1 \mathrm{~T}-\mathrm{Cu}_{x} \mathrm{TiSe}_{2}$, Phys. Rev. Lett. 118, 017002 (2017).

[11] S. Yan, D. Iaia, E. Morosan, E. Fradkin, P. Abbamonte, and V. Madhavan, Influence of Domain Walls in the Incommensurate Charge Density Wave State of cu Intercalated 1T-TiSe 2 , Phys. Rev. Lett. 118, 106405 (2017).

[12] F. J. Di Salvo, D. E. Moncton, and J. V. Waszczak, Electronic properties and superlattice formation in the semimetal $\mathrm{TiSe}_{2}$, Phys. Rev. B 14, 4321 (1976).

[13] K. Tsutsumi, X-ray-diffraction study of the periodic lattice distortion associated with a charge-density wave in $1 \mathrm{~T}-\mathrm{VSe}_{2}$, Phys. Rev. B 26, 5756 (1982).

[14] D. E. Moncton, J. D. Axe, and F. J. DiSalvo, Study of Superlattice Formation in $2 \mathrm{H}-\mathrm{NbSe}_{2}$ and $2 \mathrm{H}-\mathrm{TaSe}_{2}$ by Neutron Scattering, Phys. Rev. Lett. 34, 734 (1975).
[15] W. L. McMillan, Theory of discommensurations and the commensurate-incommensurate charge-density-wave phase transition, Phys. Rev. B 14, 1496 (1976).

[16] R. V. Coleman, B. Giambattista, P. K. Hansma, A. Johnson, W. W. McNairy, and C. G. Slough, Scanning tunnelling microscopy of charge-density waves in transition metal chalcogenides, Adv. Phys. 37, 559 (1988).

[17] W. Han, E. R. Hunt, O. Pankratov, and R. F. Frindt, Biasdependent STM images of charge-density waves on $\mathrm{TaS}_{2}$, Phys. Rev. B 50, 14746 (1994).

[18] J.-J. Kim, C. Park, and H. Olin, A study of charge-densitywaves associated with the periodic lattice distortion in $1 T-\mathrm{VSe}_{2}$ using a low temperature scanning tunneling microscope, J. Korean Phys. Soc. 31, 713 (1997).

[19] H. Fukuyama, Pinning in Peierls-Fröhlich state and conductivity, J. Phys. Soc. Jpn. 41, 513 (1976).

[20] H. Fukuyama and P. A. Lee, Dynamics of the charge-density wave. I. Impurity pinning in a single chain, Phys. Rev. B 17, 535 (1978).

[21] D. F. Agterberg and H. Tsunetsugu, Dislocations and vortices in pair-density-wave superconductors, Nat. Phys. 4, 639 (2008).

[22] Y. I. Joe, X. M. Chen, P. Ghaemi, K. D. Finkelstein, G. A. de la Peña, Y. Gan, J. C. T. Lee, S. Yuan, J. Geck, G. J. MacDougall, T. C. Chiang, S. L. Cooper, E. Fradkin, and P. Abbamonte, Emergence of charge density wave domain walls above the superconducting dome in 1T-TiSe 2 , Nat. Phys. 10, 421 (2014).

[23] L. J. Li, E. C. T. O’Farrell, K. P. Loh, G. Eda, B. Özyilmaz, and A. H. Castro Neto, Controlling many-body states by the electric-field effect in a two-dimensional material, Nature $\mathbf{5 2 9}$, 185 (2015).

[24] B. Hildebrand, T. Jaouen, C. Didiot, E. Razzoli, G. Monney, M. L. Mottas, A. Ubaldini, H. Berger, C. Barreteau, H. Beck, D. R. Bowler, and P. Aebi, Short-range phase coherence and origin of the $1 \mathrm{~T}-\mathrm{TiSe}_{2}$ charge density wave, Phys. Rev. B 93 , 125140 (2016).

[25] R. E. Thomson, B. Burk, A. Zettl, and J. Clarke, Scanning tunneling microscopy of the charge-density-wave structure in 1T-TaS 2 , Phys. Rev. B 49, 16899 (1994).

[26] A. Mesaros, K. Fujita, S. D. Edkins, M. H. Hamidian, H. Eisaki, S.-i. Uchida, J. C. S. Davis, M. J. Lawler, and E.-A. Kim, Commensurate $4 \mathrm{a}_{0}$-period charge density modulations throughout the $\mathrm{Bi}_{2} \mathrm{Sr}_{2} \mathrm{CaCu}_{2} \mathrm{O}_{8+x}$ pseudogap regime, Proc. Natl. Acad. Sci. USA 113, 12661 (2016).

[27] N. D. Mermin, The topological theory of defects in ordered media, Rev. Mod. Phys. 51, 591 (1979).

[28] C. J. Arguello, S. P. Chockalingam, E. P. Rosenthal, L. Zhao, C. Gutiérrez, J. H. Kang, W. C. Chung, R. M. Fernandes, S. Jia, A. J. Millis, R. J. Cava, and A. N. Pasupathy, Visualizing the charge density wave transition in $2 \mathrm{H}-\mathrm{NbSe}_{2}$ in real space, Phys. Rev. B 89, 235115 (2014). 